\title{
Labyrinthe
}

22 | 2005 (3)

La Biopolitique (d')après Michel Foucault

\section{Le projet de constitution européenne à la lumière de Foucault}

Éric Mollet

\section{(2) OpenEdition \\ Journals}

Édition électronique

URL : http://journals.openedition.org/labyrinthe/1039

DOI : $10.4000 /$ labyrinthe.1039

ISSN : 1950-6031

Éditeur

Hermann

Édition imprimée

Date de publication : 1 novembre 2005

Pagination : 111-117

Référence électronique

Éric Mollet, «Le projet de constitution européenne à la lumière de Foucault », Labyrinthe [En ligne],

22 | 2005 (3), mis en ligne le 22 juillet 2008, consulté le 21 avril 2019. URL : http://

journals.openedition.org/labyrinthe/1039; DOI : 10.4000/labyrinthe.1039

Propriété intellectuelle 


\title{
LE PROJET \\ DE CONSTITUTION EUROPÉENNE À LA LUMIÈRE DE FOUCAULT
}

\author{
Éric Mollet \\ ericmollet@freesurf.fr
}

Parti pour analyser la forme de gouvernementalité qui lui permettra de saisir le concept de biopolitique, Foucault s'attardera longuement dans son cours de 1979 sur le néolibéralisme qu'il décrira dans ses variantes allemande, américaine et française. Il n'est pas important ici de savoir si ce long détour par l'histoire contemporaine se soldera par un échec. Car, ce qui frappe avant tout, à la lecture de ce texte, c'est sa double actualité. Actuel, le cours de Foucault professé en 1979 l'est d'abord par les références qu'il fait à l'actualité politique immédiate, lorsqu'il est question de Raymond Barre ou de Valéry Giscard d'Estaing alors président de la République. Mais actuel, il l'est aussi, autrement. Naissance de la biopolitique a été publié en septembre 2004, soit huit mois avant le référendum pour la ratification du projet de Constitution européenne. Peu importe sa position au regard de l'ensemble de l'œuvre du philosophe, ce texte peut être utilisé comme une boîte à outils permettant non seulement de donner un éclairage particulier sur des thèmes, des éléments et des termes du projet de Constitution européenne, mais également d'y faire apparaître certaines zones d'ombre.

Ce que nous proposons ici, c'est d'utiliser le texte de Foucault et de le confronter au projet de Constitution européenne avec, en arrière fond, la question du devenir de la protection sociale en France.

Lorsqu'il analyse le néolibéralisme, Foucault prend soin de le distinguer du libéralisme classique. Pour le libéralisme du XVIII ${ }^{\mathrm{e}}$ siècle le problème était de savoir « comment, à l'intérieur d'une société politique toute donnée, on pouvait découper, ménager un espace libre qui serait celui du marché. Le problème du néolibéralisme, c'est, au contraire, de savoir comment on peut régler l'exercice global du pouvoir politique sur les principes d'une économie de marché » (NB, p. 137). Différence capi- 
tale puisque, dans un cas, il s'agit de créer un espace pour le marché à partir d'une instance politique, l'État, tandis que, dans l'autre, il s'agit d'ajuster et même d'engendrer le politique dans et par le marché. Foucault précisera d'ailleurs que contrairement au libéralisme classique qui prônait une limitation externe de l'État, le néolibéralisme se présente comme «principe fondateur et légitimant de l'État» (NB, p. 223), comme moyen de fonder un État à partir de la liberté économique (NB, p. 87-88).

Le projet de Constitution fait-il avancer d'un cran le projet néolibéral, en proposant la création d'une instance politique émanant de l'économique? L'Europe, dont l'ambition est de se construire plus réelle, plus concrète, plus forte, plus compétitive, avec pour devise, «Unie dans la diversité », affirmera-t-elle son unité au-delà des identités, des différences nationales, locales, dans et par l'économie?

On peut sans doute objecter que dans le projet de Constitution il n'est nulle part question de créer un État européen supranational ou une supernation. Construire l'Europe requiert l'invention d'une nouvelle forme de gouvernementalité telle qu'elle rende possible de subsumer, sous l'autorité légitime d'un ou plusieurs intérêts communs, des États, des pays, à la fois liés et divisés par leur histoire et leur culture. L'ambition européenne demande de se situer entre la nécessité de laisser jouer la différence entre les pays membres - en somme leur laisser une autonomie politique relative - et la nécessité de centraliser le pouvoir de manière à mener une politique commune cohérente, à orienter dans une même direction l'ensemble des intérêts de chacun dans un but commun. Comment ne pas trop gouverner pour laisser aux gouvernements locaux des prérogatives en matière de santé, de sécurité ou de gestion du territoire et de la population tout en assumant une action politique commune, déterminante et légitime?

L'idée du projet de Constitution serait alors, non pas de créer un État supranational ou une supernation, mais de fonder un système de gouvernance, à partir de la liberté économique, une gouvernementalité commune à tous les États européens. Si tel est bien le projet néolibéral, et si le projet de Constitution est d'essence néolibérale, nous devrions trouver en lui des éléments corroborant cette hypothèse. En nous reportant à l'article I-3 $\$ 3$ nous pouvons lire: «L’Union œuvre pour le développement durable de l'Europe fondé sur une croissance économique équilibrée et sur la stabilité des prix, une économie sociale de marché hautement compétitive, qui tend au plein emploi, au progrès social [...].» 
Ce qui fonde le développement de l'Europe, c'est « une croissance économique et la stabilité des prix ». Le progrès social, le plein emploi, l'économie sociale de marché sont fondés, c'est-à-dire reposent sur et dépendent de critères économiques premiers et ordonnateurs.

Si nous nous reportons aux pages de Naissance de la biopolitique où Foucault examine la question des « actions conformes » qui fixent les modalités d'actions du gouvernement libéral, nous retrouvons dans les mêmes termes les mêmes objectifs: « [...] pour les objectifs, une action régulatrice aura forcément pour objet principal la stabilité des prix [...] entendue non pas comme fixité, mais comme le contrôle de l'inflation. Et, par conséquent, tous les autres objectifs, en dehors de cette stabilité des prix, ne peuvent venir qu'en second lieu [...]. Jamais, en aucun cas, ils ne peuvent constituer un objectif premier. En particulier ne doivent pas constituer un objectif premier le maintien du pouvoir d'achat, le maintien d'un plein emploi [...]. » (P. 143-144). Pour ajouter plus loin que le plein emploi n'est pas un objectif, et que, peut-être, le chômage serait nécessaire pour l'économie.

Le plein emploi, bien que fondé sur la stabilité des prix, est présenté dans la première partie du projet de Constitution comme un objectif de l'Union pour... disparaître dans la troisième partie et être remplacé par « niveau d'emploi élevé ». À ce sujet, il faut souligner que, d'après Valéry Giscard d'Estaing, « la troisième partie n'a jamais fait l'objet d'aucune discussion de fond à la Convention puisque [ils n'avaient] pas le droit d'en modifier le contenu ${ }^{1} »$. Cependant, que cette partie ait été ou non l'objet de discussion n'explique et ne légitime en rien la contradiction. Le glissement sémantique, quelle que soit son origine, est problématique car lourd de conséquences. L'expression « tendre vers le plein emploi » est ambiguë et peut être entendue de deux façons. Soit, elle renvoie à une politique volontariste en direction de l'emploi, soit elle fait du plein emploi une des conséquences de la politique économique menée, mais certainement pas un objectif premier.

En nous reportant à l'article III-69 $\S 2$ où il est question de la politique économique et particulièrement de l'euro, nous pouvons lire que « l'objectif principal [de la politique monétaire] est la stabilité des prix et, sans préjudice de cet objectif, de soutenir les politiques économiques générales dans l'Union ». Pour confirmer cela l'article III-99 § 1 établit:

1. Voir Réflexions sur la crise de l'opinion à l'égard de l'Europe, dans Le Monde du 15 juin 2005. 
«L'Union contribue à la réalisation d'un niveau d'emploi élevé [...].» Le paragraphe 2 consacre l'expression : «L'objectif consistant à atteindre un niveau d'emploi élevé est pris en compte dans la définition et la mise en œuvre des politiques et des actions de l'Union ${ }^{2}$.» La hiérarchie stricte entre les objectifs dans un gouvernement libéral signalés par Foucault où la stabilité des prix est l'objectif premier se retrouve ici.

En s'attardant sur le glissement sémantique, d'autres éléments apparaissent. Rappelons d'abord que le plein emploi n'est pas l'absence de chômage. Il existe toujours un chômage structurel engendré par l'inadéquation de l'offre et de la demande sur le marché de l'emploi. Le plein emploi est un objectif et avant tout un objectif politique. C'est ce vers quoi tend une action politique volontariste. Par contre, le terme «niveau d'emploi élevé » n'est pas un objectif mais un effet ou la conséquence d'une économie fondée sur la stabilité des prix. Un objectif suppose que l'on prenne certaines mesures concrètes pour le remplir. Un effet, en revanche, sera toujours secondaire, de l'ordre de la conséquence, du résultat. Pour cette raison, la différence entre « plein emploi » et « niveau de travail élevé » est fondamentale. Le niveau de travail élevé est la conséquence d'une économie fondée sur la stabilité des prix et le contrôle de l'inflation: taux d'intérêts contrôlés et monnaie stable. Mais cette politique a un prix pour la population: le chômage de masse. Faire le choix du plein emploi, au contraire, c'est tout mettre en œuvre pour la création d'emplois à l'aide d'une politique volontariste, en jouant sur les taux d'intérêts et en acceptant un taux d'inflation plus important. C'est donner un but, un sens, une fin à l'activité économique, c'est orienter et diriger l'économie, c'est prendre des mesures politiques concrètes dont l'objectif sera de favoriser la création d'emplois nouveaux.

Un autre élément important apparaît dans le changement des termes. La notion de «niveau d'emploi élevé » n'a pas le caractère absolu de celle de «plein emploi ». Elle a, au contraire, une dimension éminemment relative. Dans un contexte où la population active compte plus de $10 \%$ de chômeurs, un taux de chômage à $6 \%$ constitue un niveau de travail élevé. Le caractère relatif de «niveau de travail élevé » conduit donc à la notion de « seuil ». Car, à partir du moment où l'on a fait le choix d'une politique fondée sur la stabilité des prix, il faut fixer et ajus-

2. Projet de Constitution européenne, Chap. III, Politiques dans d'autres domaines spécifiques, Section 1, Emploi. 


\section{Le projet de Constitution européenne à la lumière de Foucault}

ter les variables économiques, et déterminer ce qui est tolérable et ce qui ne l'est pas. Quand passera-t-on d'un taux de chômage normal à un chômage de masse? Quel est le rapport le plus juste entre le taux de chômage et les taux d'intérêts, par exemple?

L'enjeu sera alors de fixer la limite, le seuil, au-delà duquel la situation risque de devenir critique. Il s'agit de trouver le juste équilibre qui permet au jeu économique d'être le plus actif possible. L'action se porte non pas sur l'individu comme joueur, mais sur les règles du jeu ellesmêmes. Il faut que l'individu ait plus d'intérêt à travailler, à entreprendre, qu'à ne pas travailler. Cette idée, nous la retrouvons dans la notion « d'impôt négatif ». L'impôt négatif, nous dit Foucault, gère non pas les causes mais les effets de la pauvreté (NB, p. 210). Cette problématique de l'impôt négatif qui vient du débat américain est une solution proposée par les néolibéraux. L'enjeu est de neutraliser les effets des politiques sociales sur l'économie. Loin d'être étrangère aux débats français et européen, cette idée a été reprise, selon Foucault, dès 1972 dans l'entourage de Valéry Giscard d'Estaing (NB, p. 208). Et même si ce système de l'impôt négatif - qui consiste à assurer aux personnes dont le revenu est insuffisant un certain niveau de consommation limite tel qu'il ne devienne pas « une allocation supplémentaire comme une sorte de moyen de vivre qui leur éviterait de chercher un travail et de se réintroduire dans le jeu économique » (NB, p. 209) - n'a pas été appliqué en France, on peut néanmoins, nous dit Foucault, en voir « les linéaments dans la politique conjoncturelle de Giscard et de Barre » (NB, p. 212). Avec l'impôt négatif, nous avons « la constitution d'une politique économique qui n'est plus centrée sur le plein emploi, qui ne peut s'intégrer à l'économie générale de marché qu'en renonçant à cet objectif du plein emploi et à son instrument essentiel qu'est une croissance volontariste. On renonce donc à tout cela pour s'intégrer à une économie de marché. Mais ça implique un fonds de population flottante, un fonds de population liminaire [...] dans lequel les mécanismes d'assurance permettront à chacun de subsister [...] de telle manière qu'il pourra toujours être candidat à un emploi possible, si les conditions du marché l'exigent» (idem).

Peu importent alors les raisons pour lesquelles l'individu se trouve en dessous du seuil. Ce que doit faire la société dans ce cas, c'est l'aider à survivre, lui garantir le minimum, le temps qu'il repasse au-dessus du seuil. Outre le fait qu'une telle position tend à rabattre le chômage conjoncturel sur le chômage structurel, elle conduit aussi à mettre de côté 
« tout ce qui pourrait avoir, dans la politique sociale, des effets de redistribution générale des revenus, c'est-à-dire, en gros, tout ce qu'on pourrait placer sous le signe de la politique socialiste » $(N B$, p. 211). Plutôt que de tendre vers le plein emploi, le problème est de définir « un seuil relatif de pauvreté absolue », " c'est-à-dire ce seuil en-dessous duquel on considère que les gens n'ont pas un revenu décent susceptible de leur assurer une consommation suffisante » (idem).

Aujourd'hui, les conditions ont changé: trente ans ont passé et non seulement les mentalités se sont peu à peu habituées aux thèses et aux discours des néolibéraux, mais surtout la France est fortement impliquée dans le processus européen, et l'État français, avec ses représentants, n'est plus seul responsable de l'orientation politique du pays. L'Europe et les administrations européennes sont entrées de façon de plus en plus prégnantes dans le jeu de la politique intérieure française et des autres pays membres. Les conditions ont changé, et s'il n'était pas possible de mettre en œuvre une telle politique où l'économie et le souci de justice sociale sont hétérogènes l'un à l'autre à l'échelle française, il devient aujourd'hui possible de le faire au niveau européen.

Posons le problème autrement, en nous reportant à la question de la sécurité sociale en France. Le débat précédant le référendum a plusieurs fois abordé ce sujet, et des réponses contradictoires ont été données : dans certains cas, il a été affirmé que le système de santé est profondément remis en cause par le traité, tandis que, dans d'autres, il a été dit que les parlements des pays restaient seuls à décider en matière de santé et de justice sociale.

Si nous regardons les objectifs de l'Union au paragraphe 2 de l'article I -3, il est écrit que « l'Union offre à ses citoyennes et ses citoyens [...] un marché unique où la concurrence est libre et non faussée». Comment un système de sécurité sociale par redistribution des revenus peut-il s'accorder avec l'objectif d'une concurrence libre et non faussée? Là encore, le cours de Foucault est riche d'enseignements. L'enjeu de la sécurité sociale par redistribution se tient précisément dans ses rapports avec le jeu économique, avec la question de savoir si oui ou non, et comment, elle a une action sur l'économie. En 1945, la réponse était que la sécurité sociale ne peut pas avoir d'influence sur l'économie. Et d'ajouter que même si elle en a une, celle-ci ne peut être que positive. Trente ans plus tard, en 1976, nous dit Foucault, un rapport de l'Ena tient une position inverse: la sécurité sociale a une incidence sur l'économie 


\section{Le projet de Constitution européenne à la lumière de Foucault}

puisqu'elle augmente le coût du travail, d'où une répercussion sur la concurrence internationale et sur l'emploi, celui-ci étant plus coûteux pour l'employeur. Si la sécurité sociale a une incidence sur l'économie, alors il faut la transformer, ou, plus précisément, il faudrait comme le dit Foucault citant Valéry Giscard d'Estaing « dissocier entièrement ce qui correspond aux besoins de l'expansion économique et, d'autre part, ce qui correspond au souci de solidarité et de justice sociale» (NB, p. 206).

Dans la mesure où le système de sécurité sociale a une incidence sur l'économie en faussant la concurrence, elle entre en contradiction avec les objectifs de l'Union. Cela à deux niveaux: d'une part, de façon directe, parce qu'elle augmente le coût du travail et, d'autre part, de façon indirecte, parce que, assuré par des services publics, le système occupe un espace qui pourrait être un marché, celui de la santé.

Un mot revient souvent dans le cours de Foucault: «émergence». Telle ou telle chose "émerge » à un certain moment. Quel que soit le pourquoi, le comment, il faut l'enregistrer, constater que la chose émerge à un moment donné, c'est-à-dire apparaît et fait signe vers une réalité nouvelle. Est-ce trop dire que ce que Foucault saisit à l'occasion de son cours, à travers l'analyse du néolibéralisme sous ses différentes formes, c'est précisément l'émergence d'une nouvelle forme de gouvernementalité qui n'existe, au moment du cours, qu'à l'état disparate dans des pratiques et des théories isolées? Avec le projet de Constitution européenne (et sûrement après lui), nous voyons se construire la nouvelle forme de gouvernementalité identifiée par Foucault, reposant sur un nouveau régime de vérité « comme principe d'autolimitation du gouvernement» $(N B$, p. 21). Comment ne pas trop gouverner pour laisser se développer dans toute sa puissance le jeu économique par le moyen d'une concurrence la plus pure possible ou, pour le dire dans les termes du projet de Constitution «une concurrence libre et non faussée »? Comment maintenir une action politique sans que celle-ci ne vienne interférer sur l'économie et la fausser?

L'actualité du cours de Foucault vient de là. Il décrit l'émergence d'un nouveau régime de vérité, d'une nouvelle gouvernementalité que nous retrouvons aujourd'hui au niveau européen. 